\title{
Interacciones entre ONG, gobierno municipal y usuarios reducen la sobreexplotación del bosque, pero no la frenan: Un análisis cualitativo sobre una comunidad en Los Andes bolivianos
}

\author{
Interactions among $\mathrm{NGO}$, municipal government and users reduce the forest overexploitation, \\ but does not stop it: A qualitative analysis on a community in the Bolivian Andes
}

\author{
Ariel Isaías Ayma-Romay a,b*, Victoria Castro ${ }^{\text {b,c }}$

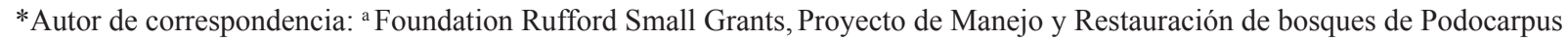 \\ y Polylepis en Independencia. Adela Zamudio \# 223. Cochabamba, Bolivia, tel: 591- 4 - 4483872, ariel.isaias.aymar@gmail.com \\ ${ }^{\mathrm{b}}$ Universidad de Chile, Facultad de Ciencias Sociales, Departamento de Antropología, Santiago, Chile. \\ c Universidad Alberto Hurtado, Facultad de Ciencias Sociales, Departamento de Antropología, Santiago, Chile.
}

\begin{abstract}
SUMMARY
The role of interactions among local organizations to improve the institutions of the communities to use forests and avoid their overexploitation is still little understood. The objective of this study was to analyze the changes of institutions and modifications of the structure of the common forest from an Andean community from Bolivia, before, during and after these interactions. A qualitative analysis was carried out with the method of thematic content (deductive and inductive). Data were managed with the Weft QDA software. Our results showed that before interacting with local organizations, the community did not have institutions to regulate forest use; thus forests were overexploited. During the interaction, the rules to use the forest and control, monitoring and sanctions systems were created and implemented. The area and other structural attributes of the forest had been maintained, though the abundance old trees of Podocarpus glomeratus had decreased. After the interactions, the rules and control system continued; however the adaptation of rules, monitoring and sanction systems have been more difficult to sustain. Therefore, it is necessary to design policies to improve the collective action among communities and local organizations to reinforce the forest community management.
\end{abstract}

Key words: policies, decentralization, common resource, Non-Governmental Organizations, municipal government.

\section{RESUMEN}

Aun se comprende poco sobre el rol de las interacciones de organizaciones locales sobre las instituciones de las comunidades para usar y evitar la sobreexplotación del bosque. El objetivo del estudio fue analizar los cambios institucionales y de la estructura del bosque de uso común de una comunidad boliviana andina, antes, durante y después de estas interacciones. El análisis fue cualitativo de tipo inductivo y deductivo con el método de análisis de contenido temático. Para el manejo de datos se utilizó el programa Weft QDA. La comunidad antes de la interacción con organizaciones locales no tenía instituciones que regulen el uso del bosque y este fue sobreexplotado. Durante la interacción, se crearon y ajustaron reglas de uso entre usuarios, se implementaron sistemas de control, monitoreo y sanción, y se llegó a mantener atributos estructurales del bosque, pero aún se redujo la abundancia de árboles maduros de Podocarpus glomeratus. Después de estas interacciones, la comunidad mantuvo reglas de uso del bosque y el sistema de autocontrol; sin embargo, la adaptación de reglas, monitoreo y sanciones fueron más dificultosas de sostener. Se debe diseñar políticas para que organizaciones locales y comunidades puedan acercarse y estimular su acción colectiva para reforzar el manejo comunitario del bosque.

Palabras clave: políticas, descentralización, recurso común, organización no gubernamental, gobierno municipal.

\section{INTRODUCCIÓN}

En Bolivia y en otros países, desde la década del 90, se ha promovido el diseño de políticas para promover el manejo comunitario de bosque (Agrawal y Gibson 1999, Pacheco 2006); sin embargo, sus resultados son heterogéneos (Andersson 2005). El manejo comunitario de bosque se fundamenta en la gestión de instituciones de uso colec- tivo, que desde una perspectiva de la economía de recursos naturales, consisten en acuerdos formales e informales de grupos humanos, como normas, prácticas y costumbres, para regular su comportamiento para usar un recurso colectivo (en este caso, bosque). Por un lado, se ha demostrado que las experiencias exitosas de manejo comunitario de bosque están relacionadas al diseño y aplicación de estas instituciones locales que, por lo general, evitan su sobre- 
explotación (Ostrom 1990, 2009). Por otro lado, también existen evidencias que no todas las comunidades llegan a contar con estas instituciones para reducir la sobreexplotación, porque tienen conflictos internos y externos con otros grupos, asimetría de poder, diferentes intereses, o no se organizan para enfrentar nuevos problemas del contexto social y ecológico y fracasan (Agrawal y Gibson 1999, Andersson 2005, Andersson y Agrawal 2011).

Se propone que estas instituciones para usar el bosque pueden mejorar con la interacción de otras organizaciones locales (gobiernos municipales, ONG y otras entidades) dispuestas a participar colectivamente en el uso del bosque (Andersson 2005, Coleman y Steed 2009, Ostrom 2009); por ejemplo, existen evidencias de que las organizaciones locales pueden reforzar las normas de uso del bosque, el control, procesos de sanción, monitoreo y solucionar conflictos entre usuarios (Berkes 2002, Moscoso 2005, Andersson 2005). Sin embargo, también pueden desfavorecer las instituciones de uso del bosque, imponiendo otras reglas de uso, desconociendo conocimientos ecológicos y costumbres de los usuarios, estableciendo sanciones poco flexibles y prohibiendo el uso de los recursos (Berkes 2002, Candela et al. 2013). Así la función de las organizaciones locales y su efecto en las instituciones de las comunidades para usar el bosque pueden ser diversos.

En Bolivia, las antiguas y actuales políticas de uso del bosque (Pacheco 2006) han sido poco ajustadas para el uso del bosque nativo de montaña y no incentivaron que entidades públicas nacionales y locales interactúen con las comunidades para conocer sus problemas de uso del bosque. Asimismo, las comunidades tienen poca motivación para acercarse a organizaciones públicas, porque los miran como corruptos, ineficientes y se sienten en riesgo de que los priven del uso del bosque; por tanto, no buscan cooperación de otros actores locales (Andersson 2005). En este contexto, existen comunidades que han usado el bosque a través de sus propias instituciones, alejados de entidades nacionales y locales; y pocas comunidades que han interactuado bajo acuerdos formales e informales con ONG locales y gobiernos municipales (Moscoso 2005). El resultado positivo o negativo de este alejamiento o acercamiento entre comunidades y organizaciones locales sobre las instituciones y la condición del bosque son poco conocidos.

El bosque nativo dominado por la conífera nativa de pino de monte (Podocarpus glomeratus D. Don) es poco frecuente en la Cordillera de Los Andes, pero tiene un alto valor para la diversidad biológica y para las comunidades campesinas (Mérida 1989, Navarro 2005). En Bolivia $P$. glomeratus es vulnerable a extinción y se distribuyen en pequeños parches de 50 a 300 hectáreas dentro territorios de comunidades campesinas que los usan colectivamente con fines domésticos o comerciales (Ayma-Romay et al. 2007). Las regulaciones de uso del bosque vigentes, por parte del Estado boliviano todavía son poco aplicables para el uso doméstico de este tipo de bosque; por lo que prácticamente ha dependido de los acuerdos de las comu- nidades y recientemente de gobiernos municipales u ONG interesados en participar en el manejo (Juarez 2009).

En la provincia Ayopaya del departamento de Cochabamba, este bosque ha sido fuertemente afectado por la quema y tala, motivando la creación de ONG y posteriormente la participación del gobierno municipal para evitar su desaparición y promover las forestaciones en interacción con algunas comunidades (GMI 2008). Localmente se tiene consenso que estas acciones han reducido la deforestación y la tala del bosque nativo, y que las instituciones de la comunidad funcionan y cumplen su objetivo de evitar la sobreexplotación (Moscoso 2005, Juarez 2009). Sin embargo, hay poca evidencia que la gestión comunitaria del bosque cumple este objetivo, y si esto se debe efectivamente a la interacción previa con organizaciones locales; ya que también podría deberse a otras causas sociales y económicas (Agrawal 2011). Por tanto, si hubo mejoras en la gestión del bosque, deberían existir cambios en las instituciones de la comunidad; además de mejoras o mantenimiento de la estructura del bosque (Gibson et al. 2005).

Los objetivos de esta investigación son: a) analizar cualitativamente el cambio de reglas de uso, control, sanciones, monitoreo y la estructura del bosque; antes, durante y después de las interacciones en la comunidad de Pajchanti, del municipio de Independencia (provincia Ayopaya) del departamento de Cochabamba, Bolivia y b) recomendar acciones para mejorar el manejo comunitario del bosque andino a nivel local.

\section{MÉTODOS}

Área de estudio. La comunidad de Pajchanti ( $17^{\circ} 5^{\prime}$ 'Sur y $66^{\circ} 49^{\prime}$ Oeste) se encuentra al noroeste del departamento de Cochabamba, Bolivia, en la cordillera Oriental de Los Andes (GMI 2008). Cuenta con formaciones de bosque de neblina dominado por P. glomeratus (Podocarpaceae) con un dosel entre 15 y $30 \mathrm{~m}$ de altura, y distribuidos en un rango altitudinal entre 2.800 y $3.500 \mathrm{~m}$ (figura 1). El área tiene un temperatura promedio de $15{ }^{\circ} \mathrm{C}$, precipitación anual de $911 \mathrm{~mm}$ (Servicio Nacional de Meteorología e Hidrología-SENAMHI, $2.760 \mathrm{~m}$ de altitud), la lluvia es en verano y la época seca en invierno (Navarro 2005). El bosque nativo está rodeado de cultivos agrícolas y pastizales de pastoreo; asimismo, existen bosque secundario y arbustales que reemplazaron bosques maduros. Actualmente el bosque es de propiedad colectiva de la comunidad y tiene 262 hectáreas (Ayma-Romay et al. 2007).

La comunidad proviene de un grupo aymara migrante del altiplano boliviano y de campesinos de otras comunidades del mismo municipio, que adquirieron terrenos agrícolas en la época de la reforma agraria de 1952 a 1960. Existieron 50 familias sin muchas variaciones a lo largo del tiempo. Desde ese entonces, existe claridad de límites. La tradición de uso y conocimientos ecológicos del bosque, al principio fue pobre, ya que ellos provienen de ecosistemas sin bosque (Veizaga 2012). 


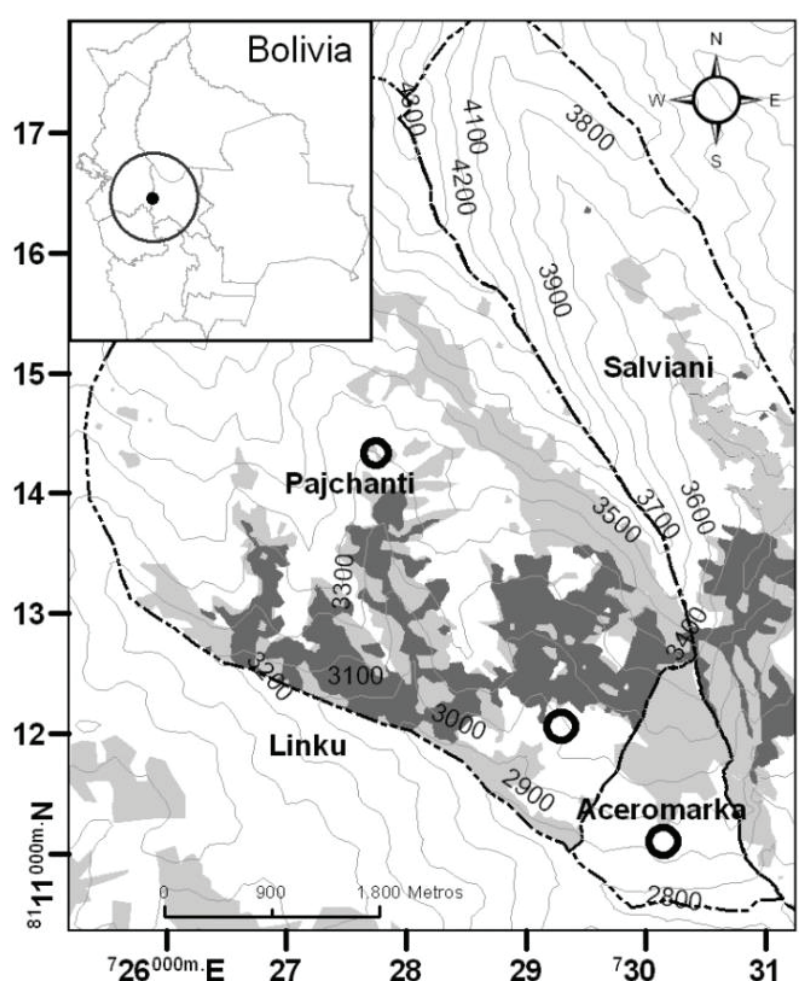

Figura 1. Área de estudio. Pajchanti y comunidades vecinas. Bosque bajo Miconia theaezans (Bonpl.) Cogn, Alnus acuminata Kunth (gris) y bosque alto P. glomeratus y Weinmannia microphylla Kunth (negro) en altitudes de 2.900 a 3.900 m. Los círculos representan centros poblados. Las líneas intermitentes son límites entre las comunidades y los círculos son áreas habitadas.

Study area, community of Pajchanti and neighbors (2,900 3,900 m a.s.l). Low forest Miconiatheaezans (Bonpl.) Cogn, Alnus acuminata Kunth (gray) and high forest $P$. glomeratus and Weinmanniami crophylla Kunth (black). Circles are areas with inhabitants and dot lines are limits between communities.

La comunidad tuvo un periodo sin interacción con organizaciones locales de 1960 a 1988. El bosque cubría gran parte de su territorio. Las organizaciones del gobierno central, ONG o gobierno municipal todavía no tuvieron presencia. La comunidad se organizó en un sindicato agrario con sus reglamentos internos, mesa directiva y asamblea comunal. El camino de Pajchanti a Palca servía para movilizarse solamente con mulas y a pie y existía un precario camino vecinal entre Palca y la capital del departamento $(220 \mathrm{~km})$ que permitía el transporte pesado. A principio y mitad de este periodo los campesinos vivieron del intercambio comercial de leña y madera (Mérida 1989, GMI 2008). También empezaron a deforestar con fines agrícolas y ganaderos y a talar los árboles más gruesos de P. glomeratus por irrisorias cantidades de dinero (Veizaga 2012).

Luego hubo un periodo de interacciones con organizaciones locales de 1989 a 2007 (ONG locales, gobierno municipal). La comunidad tuvo reuniones con los profe- sionales de las ONG, para aprobar reglas de uso del bosque (Veizaga 2012). En 1992, el gobierno municipal con la ley de participación popular, recibió por primera vez recursos económicos del gobierno central para ejecutar proyectos locales aumentando su acercamiento con las comunidades y el año 2000 creó la unidad forestal, y que tuvo eventual participación de la gestión forestal. El camino entre Pajchanti y Palca continuó precario; sin embargo, el camino vecinal que vinculaba Palca con el departamento mejoró bastante, facilitando el transporte de madera y gas para el pueblo desde la capital, lo que redujo la demanda de madera y leña de Pajchanti. La tala de árboles de P. glomeratus empezó a realizarse con motosierra, para uso doméstico y para vender (Veizaga 2012).

Después hubo un periodo sin interacción de organizaciones locales de 2008 a 2012. Las ONG finalizaron proyectos y la unidad forestal se cerró el 2010 por decisiones administrativas. En este periodo se habilitó un camino vecinal entre Pajchanti y Palca y se mejoró más aún el camino principal. El aprovechamiento de P. glomeratus continuó con motosierra, talando individuos maduros y con dimensiones suficientes ( $>60 \mathrm{~cm}$ de diámetro del tronco) para abastecer las necesidades de madera para la fabricación de muebles y madera para construcción de casas (obs. personal).

Las ONG que interactuaron con la comunidad para el manejo del bosque fueron: FUPAGEMA (Fundación para la Autogestión del Medio Ambiente) (1989 a 2003), KURMI (Desarrollo Sostenible Inter-Andino) (2004 a 2005) y un proyecto de investigación sobre manejo de $P$. glomeratus de PUMA (Fundación para la Protección del Medio Ambiente) (2006 a 2007). El gobierno municipal de Independencia (año 2000 a 2010) participó eventualmente. Estas organizaciones captaron financiamiento de agencias de cooperación internacional y no del gobierno central.

Diseño de la investigación. Se realizó un estudio de caso para analizar cualitativamente cambios de las reglas de uso del bosque, el monitoreo, el control entre miembros, las sanciones y de la estructura del bosque (superficie boscosa, densidad de árboles, área basal y composición florística) antes, durante y después de las interacciones con organizaciones locales.

Recolección de datos. Para reconstruir la situación de las instituciones de la comunidad y el bosque antes, durante y después de las interacciones con organizaciones locales, se recurrió a la narración de la gente y la revisión documental. Para tener una mejor veracidad de la información se tomaron datos de diferentes fuentes de información. Primero, se realizaron entrevistas semi-estructuradas a 25 campesinos de Pajchanti (50\% de las familias existentes), hombres, mujeres, ancianos, adultos y jóvenes; y a doce profesionales de las organizaciones locales que trabajaron con la comunidad. Las entrevistas fueron en quechua y español, según el lenguaje del entrevistado. También se obtuvieron 
datos con la técnica de observación no participativa en la comunidad durante treinta días, capturando datos en una libreta de notas, trece fotos y cinco grabaciones de video de charlas de campesinos. Además se consiguieron documentos inéditos de la comunidad (once actas de reuniones) y documentos de archivo de las organizaciones locales del municipio (cinco informes técnicos, seis cartas), una imagen satelital de 1970 (U.S. Geological Survey, Google Earth), un mapa de uso de suelo de 1987 realizado a partir de una fotografía aérea (Mérida 1989) y dos mapas de color natural compuestos de imágenes Landsat del 2007 y 2012 (U.S. Geological Survey, Earth Explorer). También se utilizaron datos cuantitativos y cualitativos de la estructura del bosque de inventarios forestales de Merida (1989) y Ayma-Romay et al. (2007). A la ausencia de datos de la estructura del bosque antes de las interacciones, se tomaron como referencia datos estructurales de áreas de bosque en la comunidad con poca intervención de tala de Ayma-Romay y Padilla (2009). También se tomaron datos de Juarez (2009) y Veizaga (2012), que hicieron estudios cualitativos sobre el uso del bosque de la comunidad con una perspectiva etnográfica. En total se tuvieron 87 documentos para analizar.

Análisis de datos. Se utilizó el método de análisis cualitativo de contenido temático, de forma inductiva y deductiva (revisar en Taylor-Powell y Renner 2003, Clark y Braun 2014). La manera inductiva fue utilizada para analizar los cambios de las instituciones y del bosque antes, durante y después de las interacciones, para buscar interpretaciones organizadas y lógicas y su significado a través de la lectura de datos narrativos (Clark y Braun 2014) y la forma deductiva, para cuantificar las percepciones de funcionamiento de las instituciones y el cambio de la extensión y abundancia de árboles maduros del bosque antes, durante y después de las interacciones (Elo y Kyngäs 2007). Para iniciar el análisis inductivo, se conformó la unidad de análisis, transcribiendo en formato de texto todas las fuentes de información (entrevistas-semiestructuradas, fotos, mapas, grabaciones, documentos, artículos) y cargándolos al programa Weft QDA (Fenton 2006). En este se generaron áreas de contenido con los periodos de análisis (antes, durante y después de interacciones) y las instituciones de la comunidad (reglas, control, sanciones, monitoreo) y de la estructura del bosque (datos cuantitativos o narraciones de la extensión, dominancia del área basal, densidad de árboles, cobertura de dosel, composición florística). A través de la lectura e interpretación, se procedió a generar códigos (conjunto de palabras) que ayudaron a capturar el significado del texto y reducir datos, estos fueron organizándose en las diferentes áreas de contenido. Las semejanzas y disimilitudes entre los códigos dentro de cada una de las áreas de contenido condujeron a crear sub-temas con nuevo significado; la comparación entre estos llegó a formar temas, y la comparación de estos produjo contenidos temáticos con las interpretaciones y explicaciones generales.
Para el análisis deductivo, en el mismo Weft QDA se cuantificó la cantidad de documentos que tenían códigos con percepciones de cambio de la extensión y la abundancia de árboles maduros agrupados en tres categorías (reduce, sin cambios, aumenta) y documentos con percepciones del funcionamiento de reglas, sanciones, control agrupado en dos categorías (bien, mal), antes durante y después de la interacción de organizaciones locales. Estas frecuencias fueron sumadas y expresadas en porcentaje ( $c f$ Elo y Kyngäs 2007).

\section{RESULTADOS}

Cambio de instituciones de la comunidad y el bosque antes, durante y después de las interacciones con organizaciones locales (según contenidos temáticos). Las interacciones cambiaron y facilitaron la implementación de instituciones de uso del bosque de la comunidad basados en tradiciones agropecuarias. Después de estas interacciones estas se han mantenido parcialmente, permitiendo desacelerar la explotación del bosque y mantener gran parte de la estructura del bosque; sin embargo, fueron parcialmente efectivas porque no fueron suficientes para evitar la pérdida de la abundancia de árboles maduros de $P$. glomeratus (la variable estructural más deteriorada) y mucho menos para mejorar la condición estructural del bosque. Los contenidos temáticos que dan cuenta de la situación de las instituciones y el bosque antes, durante y después de las interacciones se muestran a continuación (cuadro 1):

Cambios institucionales de uso del bosque (según temas y sub-temas). A continuación se describen cómo fueron los cambios en las reglas de uso, control, sanciones y monitoreo con y sin interacción de organizaciones locales:

a) Reglas. Los temas encontrados dan cuenta que las organizaciones locales facilitaron la creación y ajuste de reglas de uso del bosque que no siempre fueron adecuadas, pero sí muy consensuadas; luego de estas interacciones la comunidad mantuvo las reglas acordadas pero le costó adaptarlas (figura 2). Analizando los sub-temas, antes de las interacciones existían algunas reglas agropecuarias que redujeron indirectamente la sobreexplotación del bosque (evitando eventualmente incendios intencionados y regulando el número de ganado dentro del bosque), pero estas fueron insuficientes para evitar la tala y quema del bosque por usuarios miembros y no miembros de la comunidad (figura 2). De los nueve documentos que trataron este tema, seis sugieren un mal funcionamiento de las reglas (figura 3).

Durante la interacción de las organizaciones locales, las reglas poco a poco colocaron más restricciones para el uso del bosque (figura 2). En 1992 se prohibió la quema, tala de árboles y arbustos y el ingreso de ganado al bosque de usuarios que no pertenecían a la comunidad; así excluyeron y redujeron el número de usuarios. Luego prohibie- 
Cuadro 1. Contenidos temáticos sobre el cambio de instituciones de uso del bosque de la comunidad y los cambios estructurales del bosque antes durante y después de las interacciones locales.

Thematic contents about change of institutions and modifications of the forest structure, before, during and after interactions with local organizations.

\begin{tabular}{|c|c|c|c|}
\hline Áreas de contenido & Antes & Durante & Después \\
\hline $\begin{array}{l}\text { Cambios en } \\
\text { instituciones de la } \\
\text { comunidad }\end{array}$ & $\begin{array}{l}\text { No hubo creación de reglas, control, } \\
\text { sanciones para el uso del bosque, } \\
\text { pese a que las observaciones de los } \\
\text { campesinos sobre su perdida fueron } \\
\text { evidentes y hubo instituciones } \\
\text { agropecuarias instaladas }\end{array}$ & $\begin{array}{l}\text { Se crea, aplica y ajusta } \\
\text { reglas, sanciones y } \\
\text { predicciones sobre el bosque } \\
\text { y se motivó el control } \\
\text { basado en instituciones } \\
\text { agropecuarias }\end{array}$ & $\begin{array}{l}\text { Se mantiene reglas y control, se carece } \\
\text { de adaptación de reglas, las sanciones } \\
\text { no siempre se aplicaron por asimetrías } \\
\text { de poder y el monitoreo es postergado } \\
\text { por divergencias en la predicciones } \\
\text { futuras del bosque }\end{array}$ \\
\hline Cambios en bosque & $\begin{array}{l}\text { Se reduce la extensión del bosque, } \\
\text { la abundancia, dominancia y } \\
\text { composición de especies }\end{array}$ & $\begin{array}{l}\text { Se mantiene la extensión y } \\
\text { composición, se recupera } \\
\text { parte de la dominancia y } \\
\text { reduce la abundancia de } \\
\text { árboles maduros }\end{array}$ & $\begin{array}{l}\text { Se mantiene la extensión y otros } \\
\text { atributos estructurales y reduce la } \\
\text { abundancia de árboles maduros }\end{array}$ \\
\hline
\end{tabular}

Areas de contenido
Sub-temas

- OE y comunidad crearon, aplicaron y ajustaron reglas de uso del bosque basado en costumbres agropecuarias

- Mantención de reglas después de OE pero poca adaptación

- Los ajustes de reglas no siempre fueron adecuados para el bosque

- Control surge automáticamente basado en costumbres agropecuarias

- El control comunal se mantiene con o sin $\mathrm{OE}$

- Control de OE reforzó el control comunal.

Figura 2. Cambios en las reglas y control antes, durante y después de las interacciones de organizaciones locales (OE) (sub-temas y temas).

Changes in rules and control, before, during and after interactions with local organizations (OE) (sub-themes and themes).

ron quemar el bosque y la tala de árboles para uso de leña para todos los miembros de la comunidad, determinaron recolectar leña solamente de árboles caídos por el viento, ramas y arbustos secos y establecieron pedir permiso a la comunidad para talar árboles de $P$. glomeratus para vender o usar domésticamente. Luego el año 2003 actualizaron las reglas estableciendo algunos atributos de forma y estado de los árboles de $P$. glomeratus para ser cosechados; por ejemplo, prohibieron cortar árboles sanos, si existían árboles caídos naturalmente por el viento, o dañados con ramas partidas, secos o muy viejos. Contradictoriamente, algunos ajustes de reglas de aprovechamiento de árboles no siempre contribuyeron a reducir la explotación del bosque. Por ejemplo, desde 1997 aparte de la tala que hacían la familias para uso doméstico con autorización de la comunidad, se decidió talar cantidades fijas de madera cada año de $P$. glomeratus para que la comunidad fabrique muebles para vender. También la comunidad decidió pagar el servicio de tala y aserrado con motosierra con la tala de otro árbol para el operario y dueño de la motosierra; esto duplicó la cantidad de árboles cosechados. Para superar este problema, el año 2006 se prohibió el intercambio o co- 
mercialización de madera de $P$. glomeratus y lo destinaron sólo para uso doméstico; así dieron de baja la regla que les permitía el aprovechamiento árboles para fabricar muebles $\mathrm{y}$ venderlos, y además prohibieron pagar servicios de ase-

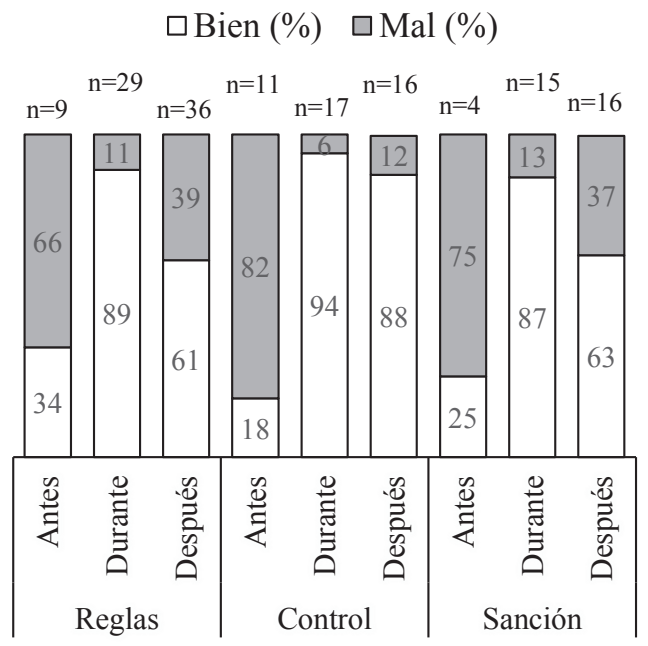

Figura 3. Percepción del funcionamiento (bien o mal) de las reglas, control y sanciones, antes, durante y después de las interacciones con organizaciones locales. Las barras muestran el porcentaje de documentos que cuentan con percepciones al respecto. $\mathrm{n}=$ número de documentos en cada categoría.

Perceptions of good or bad functioning of rules, control and sanctions, before, during and after interactions with local organizations. Bars show percentage of documents with perceptions about the theme. $n$ $=$ number of documents per category. rrío con otros árboles del bosque. En este periodo el $89 \%$ ( 25 de 29 documentos) sugirieron un buen funcionamiento de las reglas (figura 3 ).

Después de la interacción de organizaciones locales el funcionamiento las reglas se mantuvieron y aplicaron regularmente. Aunque redujo la frecuencia de documentos que consideraron su buen funcionamiento a $61 \%$ (figura 3); y aumentó a un $39 \%$ aquellos que sugieren su mal funcionamiento, debido a que algunas reglas se olvidaron (como realizar reforestaciones con árboles nativos o tratamientos silviculturales en el bosque); además no hubo ajustes o adaptaciones de consideración en las reglas para discutir recientes problemas del bosque observados por algunos miembros, como el agotamiento de árboles maduros de P. glomeratus mayores a $80 \mathrm{~cm}$ de diámetro.

b) Control. Los temas encontrados sugieren que el control surge automáticamente con la aprobación de reglas consensuadas, es reforzada con el control externo de organizaciones locales, y se mantiene con o sin interacción de estas (figura 4). Analizando los sub-temas, antes de la interacción con organizaciones locales no hubo control del bosque, porque ni los miembros, ni organizaciones locales contaban con reglas en qué apoyarse, si hubo control agropecuario y denuncias por mal uso del agua de riego, daño de cultivos agrícolas entre otros. El $82 \%$ de los documentos que tratan este tema sugiere un mal funcionamiento del control en este periodo (figura 3). Durante las interacciones con organizaciones locales, la creación de las reglas motivó que la misma comunidad controle el uso del bosque y elija un secretario de medio ambiente para res-
Áreas de contenido

Sub-temas
Temas

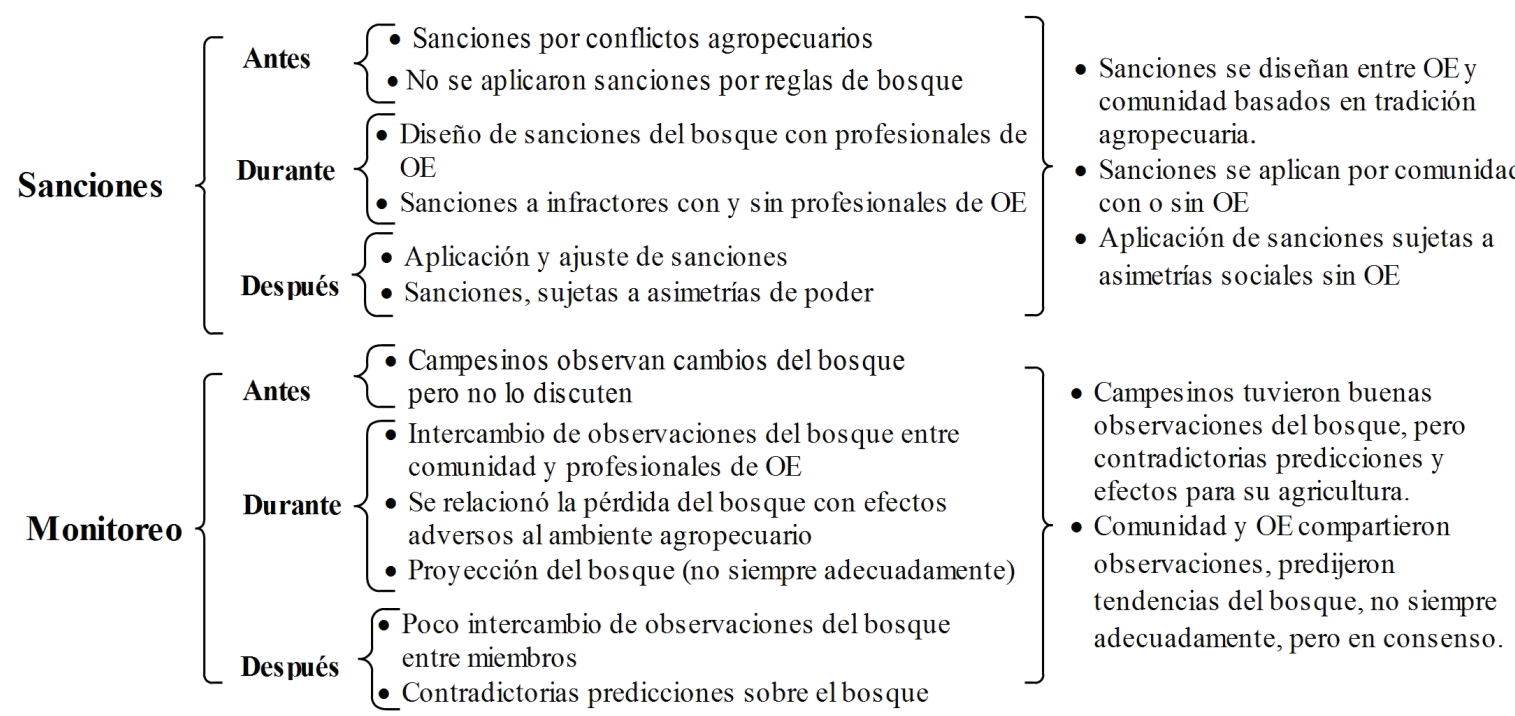

Figura 4. Cambios en las sanciones y monitoreo del bosque antes, durante y después de las interacciones de organizaciones locales (OE) (sub-temas y temas).

Changes of sanctions and monitoring before, during and after interactions with local organizations (OE) (sub-themes and themes). 
ponsabilizarse de controlar y verificar la tala de los árboles de $P$. glomeratus, según sus costumbres de control agropecuario. Inicialmente el control se concentró en excluir el uso del bosque a no miembros de la comunidad, luego el control se realizó entre ellos mismos. Este tipo de control interno se reforzó por el eventual control externo del gobierno municipal o de una ONG para dar el cumplimiento de las reglas e iniciar la denuncia; por ejemplo, un anterior técnico de la unidad forestal nos dijo "Los mismos campesinos venían a la unidad forestal para pedir autorización para talar un árbol, previa autorización de la comunidad, para no tener problemas entre nosotros" ${ }^{\prime}$. El $94 \%$ de los documentos sugirió un buen funcionamiento del control en este periodo (figura 3 ).

Después de las interacciones de las organizaciones locales, el control se mantuvo activo pese a que ya no hubo control externo del gobierno municipal, ni de las ONG. Generalmente no ha existido ningún árbol talado de $P$. glomeratus, ni incendios sin que la mayoría de los miembros lo conozcan y el secretario de medio ambiente lo informe. "Ahora ya nos controlamos más entre nosotros para la tala y seguimos controlando que nadie de otra comunidad saque nada del monte" 2 . El $88 \%$ de los documentos sugiere un buen funcionamiento del control en este periodo (figuras 2 y 3 ).

c) Sanciones. Los temas sugieren que las organizaciones locales facilitaron el diseño de sanciones y su aplicación basada en tradiciones agropecuarias de la comunidad, cuando no hubo las interacciones la comunidad se continuó aplicando, pero no en todos los casos por asimetrías de poder. Entrando a los subtemas, antes de las interacciones, la comunidad sancionaba sobre infracciones agropecuarias (conflictos de agua, daño de cultivos agrícolas), pero no existían sanciones por tala o quema del bosque, porque no habían reglas que limiten estas acciones (figura 4). Durante la interacción de organizaciones locales, se diseñó sanciones por infracciones a las reglas de bosque para miembros y no miembros de la comunidad y fueron aplicándose regularmente, previa denuncia, por iniciativa de la misma comunidad, con o sin ayuda del gobierno municipal u ONG. En este periodo diez sanciones provienen de quemas accidentales del bosque y pastizales y dos sanciones por tala no autorizada de $P$. glomeratus. Por ejemplo, un campesino recordó: "Una vez don Julio quemó el bosque sin querer al habilitar su barbecho, nos reunimos y justo vino el responsable de la alcaldía, hablamos y decidimos sancionarle con una multa de 500 bolivianos y plantar árboles nativos y eucaliptos para reparar el daño (...."’3. Las infracciones fueron resueltas con sanciones flexibles, que no necesariamente consistieron en multas económicas, como establecían las reglas, pero igual fueron consensuadas según cada circuns-

\footnotetext{
F Fernández, comunicación personal, 15 de marzo, 2012.

C Mamani, comunicación personal, 29 de enero de 2011.

M Calani, comunicación personal, 21 de enero, 2011.
}

tancia. Un campesino comentó: "Don Justo taló un árbol de pino de monte para hacer su casa (...) sin autorización del sindicato y la comunidad no le dejo sacarlo. Él se enojó, pero esa fue su sanción por desobedecer"4. El $87 \%$ de los documentos sugirió para este periodo un buen funcionamiento de las sanciones (figura 3 ).

Después de las interacciones de organizaciones locales, las sanciones han continuado efectuándose por iniciativa de los usuarios. Hubo tres casos de sanciones por quemar accidentalmente pequeñas partes de borde del bosque $\left(<300 \mathrm{~m}^{2}\right)$ y una sola sanción por talar más árboles de lo autorizado por la comunidad. Muchas de las sanciones que debían ser pagadas en efectivo se cumplieron con trabajo comunitario (plantación de árboles exóticos). Sin embargo, la aplicación de sanciones no se efectuaron para dos infractores con más poder en la comunidad, que talaron más árboles de lo autorizado y no fueron denunciados ni sancionados pese a que todos conocían el hecho. Un campesino manifestó "el mismo secretario de medio ambiente ha sacado tanta madera para la casa de su hermano, se aprovechó de su cargo, fue abusivo"s (figura 4). En este periodo, redujeron los documentos con percepciones de buen funcionamiento de las sanciones a $62 \%$ respecto al periodo anterior (figura 3).

d) Monitoreo del bosque. Los temas hallados sugieren que las organizaciones facilitaron compartir observaciones del bosque y a proyectar tendencias de su estructura y sus efectos para su agricultura, pero no siempre fueron adecuadas (figura 4). Entrando a analizar los sub-temas, antes de la interacción de organizaciones, todos los usuarios y otros profesionales observaron que el bosque declinaba aceleradamente en superficie y que la densidad de árboles maduros disminuía junto con otros atributos; sin embargo, la gente continuó talando y quemando, y no intercambiaron estas observaciones en su comunidad para generar reglas (figura 4). Durante la interacción, a través de reuniones se intercambiaron las observaciones de la comunidad y de los profesionales de ONG (a través de mapas de cambio de uso de suelo, inventarios forestales y otras observaciones de campo) y permitió que surjan en consenso proyecciones intuitivas sobre la pérdida de la superficie boscosa, la abundancia de árboles de $P$. glomeratus y sus efectos para su vida como la disminución de leña, agua, madera entre otros. Estas observaciones fueron importantes para ajustar reglas de uso del bosque, pero no necesariamente fueron adecuadas para evitar la sobreexplotación, porque fue difícil predecir la tendencia de la densidad de árboles maduros, incluyendo sus cosechas; sin embargo, fueron consensuadas. La percepción de los cambios de la abundancia de árboles durante las interacciones fue lo más controversial, lo que coincide con desatinos en el ajuste de reglas y en las cosechas (figura 5).

\footnotetext{
J Cáceres, comunicación personal, 18 de febrero, 2011.

R Ayala, comunicación personal, 23 de enero de 2011
} 


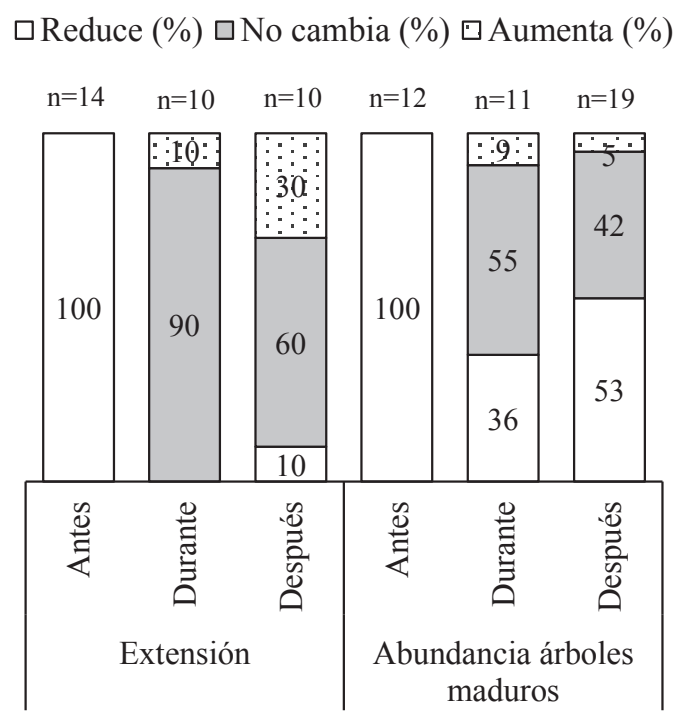

Figura 5. Percepción de cambio de la extensión y abundancia de árboles maduros del bosque antes, durante y después de las interacciones con organizaciones locales.

Perceptions of change about area and abundance of old tree before, during and after local interactions.

Después de estas interacciones, los miembros continuaron monitoreando el bosque; sin embargo, ellos no discutieron sus observaciones o proyecciones futuras sobre el bosque. Además continuaron las controversiales proyecciones de la densidad de árboles maduros de P. glomeratus. El $53 \%$ de los documentos sugirió que la abundancia de árboles maduros no ha cambiado, pero el $42 \%$ indicó que se ha reducido incluso cumpliendo las reglas acordadas (figura 5).

El bosque antes, durante y después a la interacción de organizaciones locales. Antes de las interacciones de organizaciones locales, el bosque redujo fuertemente en su estructura. El $100 \%$ de los documentos que trataron este tema coincidieron que la extensión y abundancia de árboles maduros redujeron (figura 5). Comparando mapas e informes, la extensión se redujo $(\approx 35 \%$, de 400 a 260 ha) principalmente antes de 1970, porque desde entonces hasta el 2007 la extensión no cambió sustancialmente. Además los datos de inventarios forestales sugieren que la dominancia también redujo $\left(\approx 18 \mathrm{~m}^{2} \mathrm{ha}^{-1}\right.$, de 36 hasta $14-16 \mathrm{~m}^{2}$ ha $^{-1}$ ); asimismo, la densidad de árboles mayores a $10 \mathrm{~cm}$ de DAP, de $\approx 500$ árboles ha ${ }^{-1} \mathrm{a} \approx 430$ árboles ha ${ }^{-1}$. Según relatos de campesinos y profesionales, en este periodo desaparecieron los árboles más gruesos que alcanzaban incluso $200 \mathrm{~cm}$ de DAP. Asimismo, el bosque maduro previó a la explotación dominado en orden de importancia por P. glomeratus, Weinmannia microphylla Ruiz et Pav., Blepharocalyx salicifolius Kunth O. Berg, Cedrela lilloi C. DC, Trichilia hirta L. y Myrcianthes osteomeloides (Rusby) McVaugh cambio su composición florística por la tala de $P$. glomeratus, $C$. lilloi y $T$. hirta y la apertura de claros de dosel que permitieron el reclutamiento y crecimiento de especies arbóreas sombra-no tolerantes como M. theaezans y A. acuminata que fueron las especies más importantes luego de $P$. glomeratus y W. microphylla.

Durante la interacción con organizaciones locales, la densidad de árboles mayores a $10 \mathrm{~cm}$ de DAP no mostró cambios $\left(\mathrm{de} \approx 432 \mathrm{a} \approx 428\right.$ árboles ha $\left.^{-1}\right)$ y la dominancia incrementó ligeramente $\left(\approx 22 \%\right.$, de 14 a $\left.18 \mathrm{~m}^{2} \mathrm{ha}^{-1}\right)$. La composición florística no cambió del periodo anterior, porque siguieron dominando $P$. glomeratus, W. microphylla y se consolidaron especies sombra-intolerantes como M. theaezans, arbustos y grámineas como Chusquea sp. La abundancia de árboles maduros de $P$. glomeratus redujo antes y durante las interacciones; comparando un área pequeña de bosque maduro de la comunidad sin intervención de tala tuvo $\approx 32$ árboles ha $^{-1}$ con DAP $>60 \mathrm{~cm}$, pero al final de las interacciones con organizaciones locales, gran parte de las áreas de bosque tuvieron $\approx 10$ árboles ha- ${ }^{-1}$ con DAP $>60 \mathrm{~cm}$.

Después de las interacciones, la comparación de mapas del año 2007 y del 2012 sugieren que la extensión del bosque no cambio; aunque el $30 \%$ de los documentos sugieren que el área de bosque ha incrementado y que la abundancia de los árboles maduros redujeron en este último periodo (figura 5). Observando el bosque se constató que las especies arbóreas todavía dominantes son $P$. glomeratus, W. microphylla y M. theaezans. Para este periodo, no se contó con información de inventarios forestales recientes.

\section{DISCUSIÓN}

Interacciones de organizaciones locales, instituciones de la comunidad y bosque. Las organizaciones locales tuvieron influencia en las instituciones de uso del bosque de la comunidad, y también en la estructura del bosque, así como en otros estudios (Berkes 2002, Moscoso 2005, Andersson 2005). Aunque se ha conseguido que gran parte de los atributos estructurales del bosque se mantengan durante y después de las interacciones, todavía es cuestionable la reducción de la abundancia de árboles maduros de P. glomeratus, la especie más importante ecológica y socialmente en este tipo de bosque (Ayma-Romay et al. 2007) aún con la voluntad de la comunidad para dar cumplimiento a las reglas, el control y la aplicación de sanciones.

Antes de las interacciones con organizaciones locales, al igual que en otros estudios, el principal problema fue la ausencia de reglas de uso colectivo del bosque para regular la libre explotación entre miembros de la comunidad y de otros usuarios ajenos (Moscoso 2005), lo que llevó a no tener control, sanciones, pese a que existían observaciones de declinación del bosque. Esto puede deberse al poco capital social existente en la comunidad en ese entonces para usar el bosque (Ostrom 2009), ya que fue una comunidad migrante de un ecosistema sin bosque (Veizaga 2012). Después que se crearon y ajustaron las reglas con las orga- 
nizaciones locales, las limitaciones para llevarlas adelante fueron la poca habilidad de los usuarios para hacer ajustes de los acuerdos, sancionar a todos los infractores por las asimetrías de poder y diferentes intereses, así como en otros estudios (Andersson y Agrawal 2011), intercambiar observaciones y anticipar las tendencias futuras del bosque considerando sus cosechas (Ostrom 1990, 2009). Estas limitaciones fueron aminoradas por otras organizaciones locales cuando ejercieron funciones de control, monitoreo y facilitación de acuerdos entre miembros, pero no totalmente solucionadas.

Creación, aplicación y adaptación de reglas. En este estudio de caso, algo que facilitó la creación de reglas, fue la decisión de la comunidad para excluir a usuarios externos que usaban el bosque sin su consentimiento y el respeto a su autonomía a tomar decisiones sobre el bosque (Ostrom 1990). Decisión que en otras comunidades de la zona andina de Bolivia estuvo motivada por fuertes aspiraciones de consolidación de los derechos de propiedad del territorio (Moscoso 2005). La creación de reglas aprobadas por la misma comunidad inició procesos de control, monitoreo, sanciones y a reducir la sobreexplotación del bosque como en otros estudios que consideran que la creación de reglas es fundamental para el manejo comunitario del bosque exitoso (Ostrom 1990, Gibson et al. 2004). Las interacciones con organizaciones locales tuvieron mucha importancia al inicio de la aplicación de reglas, porque ellos ayudaron a denunciar, sancionar y mediar conflictos entre los usuarios; después se advierte que la comunidad quedó comprometida a seguir cumpliendo las reglas, como en otros estudios donde grupos humanos siguieron automáticamente las reglas creadas colectivamente, una vez que algunos empezaron a cumplirlas (Gibson et al. 2004).

Se ha demostrado que las reglas diseñadas por los mismos miembros, pueden ser más efectivas que reglas impuestas por entidades externas, debido a su fácil comprensión y debate previo entre miembros (Ostrom 1990); algo que no ocurre con reglas de gobiernos nacionales, municipales u ONG que prohíben el uso de ciertos recursos del bosque sin consultar a los usuarios (Candela et al. 2013). Sin embargo, la poca habilidad de los miembros para adaptar las reglas, cuando no interactúan con organizaciones locales puede ser un problema. Estudios previos sugieren que cambiar reglas no es nada sencillo, porque implica reorganizar los beneficios del bosque para los miembros, en donde algunos ganan y otros pierden, así algunos grupos se oponen a estos cambios; además, para adaptar una regla de uso, ellos deben advertir algún cambio en la abundancia del recurso colectivamente; al no advertirlas no deciden ajustes porque son calificadas innecesarias (Fréchete y Lewis 2011) y justamente esto es lo que más le ha costado a la comunidad después de las interacciones.

Control. El control de profesionales de las organizaciones locales sobre el bosque han complementado el control in- terno de la comunidad; este ha surgido automáticamente luego de la aprobación de las reglas de uso del bosque, porque no fueron diseñadas explícitamente, pero aún así han sido las que mejor han funcionado, como en otros estudios que sugieren que cuando los miembros diseñan, aprueban y ajustan sus propias reglas de uso, se incrementa su motivación para controlarse unos a otros y observar los cambios en el bosque automáticamente (Gibson et al. 2005). Esto también es posible porque comunidades con tradición de monitorear y controlar otros recursos comunes reducen el costo organizativo para ampliar estas funciones sobre otros recursos, como el bosque (Coleman y Steed 2013) y en la comunidad ya existía la tradición de controlar y monitorear el uso de sistemas agropecuarios. Además, porque los miembros pueden observar el comportamiento de otros miembros dentro de sus rutinas diarias con un bajo costo de tiempo y energía y sin adicionar más esfuerzo (Ostrom 1990). Por último, el control externo de una organización local, aunque eventual, pudo reducir la tentación de algunos miembros de la comunidad y otros ajenos a evadir las reglas, una conducta que ha sido también observada en otros estudios (Ostrom 1990).

Monitoreo. El monitoreo de algunos atributos estructurales del bosque con organismos sésiles como los árboles, que ocupan pequeña extensión y de fácil acceso, como en el caso de este estudio, debería ser sencillo para las comunidades, comparado con otros recursos que son más complejos de monitorear como organismos móviles por ejemplo, peces u otros recursos de más grande extensión, que reduce la capacidad de los usuarios para observarlo (Ostrom 2009); sin embargo, compartir observaciones entre usuarios y hacer proyecciones consensuadas sobre la abundancia de árboles maduros, incluyendo cosechas fue uno de las más fuertes limitaciones para evitar la sobreexplotación de $P$. glomeratus incluso para las organizaciones locales; ya que posiblemente no sea tan sencillo monitorear el cambio de la abundancia de los árboles de lento crecimiento y de longeva vida, cuyos ciclos de vida superan la expectativa de vida de los mismos usuarios y que difícilmente pueden proyectar (Ayma-Romay et al. 2007). Aún si los advirtieran, si no las comparten, no hacen proyecciones consensuadas y no toman decisiones sobre el destino de los recursos, estas no sirven para formular otras reglas de uso. Esto es importante porque si no se implementan sistemas efectivos de monitoreo, las reglas pueden estarse cumpliendo, pero con la incertidumbre que las unidades de cosecha sigan superando la capacidad del bosque para reponerlas (Gibson et al. 2005).

Sanciones. Similar al caso de estudio, se ha observado que es importante fomentar que los mismos miembros establezcan y apliquen sus propias sanciones, porque esto aumenta la probabilidad de que ellos mismos se denuncien y sancionen (Gibson et al. 2005). En el caso de estudio, las sanciones siempre fueron flexibles y adaptables al contexto, algo 
que es muy conveniente en contraposición a sanciones rígidas y fuertes que pueden desmotivar el cumplimiento de reglas (Ostrom 1990). También se debe prever que la comunidad no siempre llegó a sancionar a los infractores, causando disconformidad, ya que algunas veces miembros con más poder no fueron sancionados con la misma rigurosidad que otros ni tampoco denunciados. $\mathrm{Al}$ respecto, en otros estudios se ha observado que algunos miembros con poder se corrompen para obtener mayor beneficio. Este es uno de los principales problemas del manejo comunitario del bosque (Andersson y Agrawal 2011) y es probable que la presencia de profesionales de organizaciones locales en las reuniones de la comunidades mejoren el curso de las decisiones de la comunidad.

Recomendaciones. Las políticas deben incentivar procesos donde las comunidades creen, evalúen y adapten reglas de uso del bosque en cooperación con organizaciones locales que actúen como mediadores en el proceso de ajuste y aplicación de sanciones y reglas; y comprometerse con la realización de algunas funciones como de control y monitoreo. Para esto, es necesario ganar la confianza de las comunidades, comprender mejor sus problemas y cómo funcionan las instituciones comunales, que según Juárez (2009) es un proceso de aprendizaje continuo desde sus capacidades sociales y que es abierto a la adaptación y la incorporación de nuevos conocimientos.

Por otro lado, es crucial crear sistemas de control y monitoreo compartido entre organizaciones locales y comunidades, basados en reglas de uso aceptadas por los usuarios; estos sistemas pueden ser muy fáciles de implementar con bajo costo para ambas partes. Esto se mejoraría, estableciendo funciones y mecanismos para controlar y hacer conocer las denuncias, intercambiando observaciones e implementando sistemas simples de monitoreo de algunos atributos del bosque a través de la observación, medición y análisis de estos por la misma comunidad (principalmente la abundancia de árboles maduros que se cosechan y su crecimiento).

Es necesario que los procesos de denuncia y sanción en la comunidad sean supervisadas por veedores externos del gobierno municipal o de ONG, que pueden participar de las reuniones donde se toman las decisiones. Esto evitará que miembros con poder y diferentes intereses de la comunidad no sean sancionados.

Las organizaciones locales que trabajaron con comunidades campesinas en manejo de bosque han priorizado hasta el momento la capacitación, la creación y ajuste de reglas; sin embargo, los sistemas de monitoreo, sanción y control (interno y externo) han sido descuidados. Esto requiere compromisos a largo plazo entre ambas partes, y esto es una fuerte limitante para las ONG que no pueden sostener la continuidad de sus actividades y gobiernos municipales que no tienen definido sus políticas para conservar el bosque y son altamente dependientes de la voluntad de sus autoridades y profesionales de turno.

\section{AGRADECIMIENTOS}

Agradecemos a la comunidad de Pajchanti por hablarnos de su vida y el bosque y a los profesionales de organizaciones locales que nos contaron sus experiencias, principalmente a Grover Lisperguer. A Elsa Padilla Barroso por ayudar con las entrevistas en quechua, la traducción al español y su transcripción del material de audio, video y entrevistas. A la Agencia Cooperación Internacional de Chile por financiar parte de la investigación de la tesis de maestría y al editor y revisores anónimos de la revista que gentilmente revisaron el manuscrito e hicieron valiosos aportes para mejorarlo.

\section{REFERENCIAS}

Agrawal A. 2001. Common property institutions and sustainable governance of resource. World Development 29:16491672. DOI: 10.1016/S0305-750X(01)00063-8.

Agrawal A, C Gibson. 1999. Enchantment and disenchantment: The role of community in natural resource conservation. World Development 27:629-649. DOI: 10.1016/S0305750X(98)00161-2.

Andersson K. 2005. ¿Cómo hacer funcionar la gestión forestal descentralizada? La Paz, Bolivia. Plural. 177 p.

Andersson K, A Agrawal. 2011. Inequalities, institutions and forest commons. Global Environmental Change 21(3): 866875. DOI:10.1016/j.gloenvcha.2011.03.004.

Ayma-Romay AI, E Padilla-Barroso, E Calani. 2007. Estructura, composición y regeneración de un bosque de neblina: sugerencias silviculturales para Podocarpus glomeratus en la comunidad de Pajchanti. Revista Boliviana de Ecología y Conservación Ambiental 21:27-42.

Ayma-Romay AI, E. Padilla. 2009. Efecto de la tala de Podocarpus glomeratus (Podocarpaceae) sobre la estructura de un bosque de neblina de los Andes (Cochabamba, Bolivia). Revista Peruana de Biología 16(1): 73-79.

Berkes F. 2002. Cross-scale institutional linkages: perspectives from the bottom up. In Ostrom ET, T Dietz, N Dolsak, PC Stern, S Stonich, EU Weber eds. The drama of the commons. Washington, DC. National Academy Press. p. 293321.

Candela SE, D Stolan, E Somarilba, M Villalobos. 2013. Formas y efectos de la gobernanza forestal en los territorios indígenas bribri y cabécar de Alta Talamanca, Costa Rica. Agroforesteria de las Américas 49: 85-92.

Clark V, V Braun. 2014. Thematic analysis. In Teo T ed. Encyclopedia of critical psychology. New York, USA. Springer. p. 1947-1952. DOI 10.1007/978-1-4614-5583-7

Coleman EA, B Steed. 2009. Monitoring and sanctioning in the commons: An application to forestry. Ecological Economics 68: 2106-2113. DOI:10.1016/j.ecolecon.2009.02.006

Elo S, H Kyngäs. 2007. The qualitative content analysis process. Journal of Advanced Nursing 62: 107-115. DOI: 10.1111/j.1365-2648.2007.04569.x

Fenton A. 2006. Weft QDA for qualitative data analysis. Consultado 5 ago. 2012. Disponible en http://www.pressure.to/ qda/doc/wefthelp.pdf

Fréchette A, N Lewis. 2011. Pushing the boundaries of conventional forest policy research: Analyzing institutional change 
at multiple levels. Forest Policy and Economics 13: 582589. DOI: 10.1016/j.forpol.2011.06.012.

Gibson CC, JT Williams, E Ostrom. 2005. Local enforcement and better forests. World Development 33(2): 273-284. DOI: 10.1016/j.worlddev.2004.07.013.

GMI (Gobierno Municipal de Independencia, BO). 2008. Plan de desarrollo municipal de Independencia ajustado 2008 2012. Cochabamba, Bolivia. 175 p.

Juarez R. 2009. Normas comunales para el uso y acceso a los bosques nativos andinos en la comunidad de Pajchanti, Cochabamba, Bolivia. Acta Nova 4: 362-374.

Merida G. 1989. Evaluación de recursos y lineamientos para la restauración y uso sostenido de un bosque de neblina (Ayopaya Cochabamba, Bolivia). Tesis Ingeniero Forestal. Lima, Perú. Facultad de Agronomía. Universidad Agraria La Molina. 250 p.

Moscoso R. 2005. Normas comunales para el manejo de la vegetación nativa. Marco teórico conceptual desde la experiencia de comunidades andinas. La Paz, Bolivia. Sagitario. 56 p.

Navarro G. 2005. Provincia biogeográfica de los yungas. In Na- varro G, M Maldonado eds. Geografía ecológica de Bolivia. Santa Cruz, Bolivia. Centro de Ecología Difusión Simón I. Patiño. p. 279- 339.

Ostrom E. 1990. Governing the commons: the evolution of institutions for collective action. Cambridge, Reino Unido. Cambridge University Press. 302 p.

Ostrom E. 2009. A general framework for analyzing sustainability of social-ecological systems. Science 325:419-422. DOI: 10.1126/science.1172133.

Pacheco P. 2006. Descentralización forestal en Bolivia: implicaciones en el gobierno de los recursos forestales y el bienestar de los grupos marginados. La Paz, Bolivia. Plural. 144 p.

Taylor-Powell E, M Renner. 2003. Analyzing qualitative data. University of Wisconsin. Consultado 12 dic. 2011. Disponible en http://learningstore.uwex.edu/assets/pdfs/g3658-12.pdf

Veizaga, G. 2012. Caracterización de las presiones antropogénicas en el bosque de pino de monte (Podocarpus glomeratus) de la comunidad de Pajchanti, provincia Ayopaya. Tesis Ingeniero Forestal. Cochabamba, Bolivia. Facultad de Agronomía. Universidad Mayor de San Simón. 53 p.

Recibido: 01.12.14

Aceptado: 16.11 .15 
